\title{
Towards early detection of pulmonary hypertension: a call to arms
}

\author{
Kenneth Whyte
}

Affiliation: University of Auckland and Greenlane Respiratory Service, Auckland City Hospital, Auckland, New Zealand.

Correspondence: K. Whyte, University of Auckland and Greenlane Respiratory Service, Auckland City Hospital, Private Bag 92024, Grafton, Auckland, New Zealand. E-mail: Kenwdaadhb.govt.nz

0 @ERSpublications

The imperative over the coming decade is to improve our ability to diagnose pulmonary hypertension early http://ow.ly/pUM2Y

The more powerful the obstacle, the more glory we have in overcoming it

- Mascarille, Molière, L'Étourdi ou Les Contretemps, Act V, Scene XI

Emerging evidence demonstrates that early detection alters outcomes in systemic sclerosis-related pulmonary arterial hypertension $(\mathrm{PAH})[1]$. The endeavours of the pulmonary hypertension community over the last 20 years have improved survival with modern treatment strategies but prognosis remains poor [2].

On average, even in the current era, patients have been symptomatic for $>2$ years by the time the diagnosis of pulmonary hypertension is made $[3,4]$. If our understanding of the pathobiology of PAH is correct, then by the time of diagnosis, there will have been extensive remodelling and/or obliteration of a very significant proportion of the small muscular pulmonary arteries in our patients. The imperative over the coming decade for those of us treating pulmonary hypertension is to improve our ability to diagnose it early, when permanent damage to the pulmonary circulation is limited, leading to improved outcomes and, potentially, a normal quality of life with modern therapy.

In response to these facts, patient-led support groups in Europe, North America and Australia have instigated campaigns not only to raise awareness of the existence of PAH but also to encourage individuals with unexplained breathlessness or fatigue to pursue a diagnosis with their doctors. This will inevitably lead to increasing referrals to pulmonary vascular clinics of patients with unexplained dyspnoea, syncope or fatigue.

In some of these individuals, investigation will confirm a diagnosis of established PAH and, in others, alternative causes of their symptoms will be diagnosed. However, clinicians will be faced with symptomatic patients who have a moderate-to-high pre-test probability of pulmonary vascular disease based on clinical history or examination in whom echocardiography and other assessments at rest are unremarkable, and resting mean pulmonary artery pressure (mPAP) is normal or "borderline" at right heart catheterisation. How can we improve detection of early pulmonary vascular disease when such patients present to our clinics?

Currently, we have no proven investigative strategy to identify which of these patients have early pulmonary vascular disease and who would potentially benefit from early treatment. As not all subjects with indeterminate initial investigations can be sent to large national or supraregional centres, such an investigative strategy must be applicable locally, or at least regionally, to be effective in leading to an earlier diagnosis of PAH.

What tools should we use to investigate further such individuals? Exercise "stress" testing during right heart catheterisation was the next investigative tool in a limited number of specialised centres until it was recognised at the Fourth World Symposium on Pulmonary Hypertension in Dana Point, CA, USA, that

Received: April 162013 | Accepted after revision: Sept 032013

Conflict of interest: None declared.

Copyright @ERS 2014 
exercise pulmonary haemodynamic responses were poorly categorised and that there was no clear "normal range" of pulmonary artery pressure (PAP) responses [5]. However, exercise continues to appeal as the most logical approach, as it is during the stress of exercise that patients report their symptoms, whether they are breathlessness, excessive fatigue or, less commonly, syncope, and exercise will lead to pulmonary hypertension in the diseased pulmonary arterial bed [6]. The use of repeated haemodynamic measurements as cardiac output $(Q)$ increases during a progressive exercise test allows a PAP-Q slope to be measured and the limited data currently available suggest that, in the normal pulmonary circulation, across a range of ages, the slope is $<3 \mathrm{mmHg} \cdot \mathrm{L}^{-1}$; further data in normal populations may validate the diagnostic utility of this measurement [6].

A report in a previous issue of the European Respiratory Journal by PAVELESCU et al. [7] is thought provoking for a number of reasons in regard to detecting abnormal pulmonary vascular responses. Those authors demonstrated abnormal pulmonary haemodynamic responses during exercise stress echocardiography and during a 2-h period of chronic hypoxia in asymptomatic carriers of BMPR2 mutations. Chronic hypoxia could have limitations as a widely applicable screening test but exercise stress Doppler echocardiography, a test often felt to be technically challenging and inconsistent, proved its ability to discriminate from normal.

That report was based on participants at a single centre who were subjects in a larger multicentre study of PAP responses to exercise and hypoxia in relatives of patients with idiopathic and familial PAH [8]. Clearly, these would be core groups of individuals likely to be investigated for evidence of early pulmonary vascular disease, so it is a highly relevant study.

This research group went beyond the PAP response during exercise echocardiography and demonstrated they can measure the distensibility quotient $(\alpha)$ of the pulmonary circulation using exercise echocardiography. Briefly, mPAP, left atrial pressure and $Q$ are calculated at each workload during a progressive exercise protocol, which allows a multipoint $\mathrm{mPAP}-\mathrm{Q}$ relationship to be determined. The curvilinearity of this relationship defines the $\alpha$ of the pulmonary resistive vessels [6]. Only age, chronic hypoxia and pulmonary vascular disease have been shown to alter the $\alpha$ of the human pulmonary circulation $[6,9]$. Such a direct measurement of pulmonary vascular response during stress is potentially an ideal method of detecting early pulmonary vascular disease and this group has already reported that these measurements are repeatable [10]. Previously, $\alpha$ has been derived from cardiac magnetic resonance imaging measurements but this is complex, expensive, technically demanding and limited to a very few research centres [11].

This observation of abnormal distensibility in subjects with this genotype does not prove the presence of pulmonary vascular disease and this maybe an intermediate phenotype. However, this report should encourage further exploration of this potential marker, initially in patients with early proven pulmonary vascular disease, to determine its sensitivity, then in high-risk groups under regular "screening" for pulmonary vascular disease (e.g. patients with systemic sclerosis), to determine its potential utility.

An obvious concern is that the data of PAVELESCU et al. [7] are from a highly experienced, dedicated pulmonary vascular research unit, and that this methodology would not be "exportable" to smaller centres with more limited resources and expertise. This is a valid concern, yet the original multicentre study confirmed that, with motivated centres and standardised methodology, high-quality results can be obtained using exercise stress echocardiography. In the original multicentre study, each centre followed a standard methodology, echocardiographers were trained rigorously in the technique and interpretation of the data was carried out at a single institution producing high-quality data [8]. Results were reproducible and consistent between centres with no evidence that any centre had "aberrant" results [8].

The derivation of $\alpha$ required additional measurements [7] and we would need to ensure that this quality of measurements can be consistently reproduced in other centres as part of the proof of concept.

The challenges that the pulmonary hypertension community would face in developing and establishing this or any other potential tool for early diagnosis of $\mathrm{PAH}$ across networks of centres of varying size and expertise would be similar. The first crucial broader step that has to be taken by the pulmonary hypertension community is to standardise methodology, patterns of exercise if the stress is exercise, exact measurements to be made and quality control during acquisition of those measurements to ensure validity of the measurements in each subject during the test. This would require a working group to set and validate such standards.

Potentially, if we were to confirm the utility of this measurement $(\alpha)$ or any alternative echocardiographic measurement during exercise, we would need to involve the manufacturers of echocardiography equipment to ensure their equipment is equal to the task, that the software to make the measurements is available on their machines and that there is feedback to the echocardiographer on the quality of signals being obtained during 
the test. This is not an unrealistic expectation. Recently, a UK working party has recommended a standardised approach for echocardiographic right ventricular and pulmonary haemodynamic measurements at rest [12], which is a welcome first step for our field.

To ensure access for patients in local or regional centres, central training of staff at larger centres may be required with subsequent feedback on each individual echocardiographer's performance using a portfolio or similar approach, with oversight being provided by larger supraregional or national centres. Interpretation of results, at least for smaller centres, would logically be through more experienced larger centres, which could also monitor quality issues.

Is this a realistic approach? This specific diagnostic tool, echocardiographic determination of distensibility during exercise, is clearly, as yet, unproven. We would need to determine normal ranges in various groups (e.g. the elderly or obese), and determine sensitivity and specificity from using a standardised investigative protocol.

We need to continue to explore aggressively the value of a variety of other possible tools, including novel measurements at rest and during invasive exercise stress haemodynamic testing, as recently argued elegantly by SAGGAR and SiTBON [13]. We should not prematurely abandon a tool (exercise haemodynamics) that has a track record of providing relevant results.

In addition, there are a number of other potential investigative tools that should be explored in parallel studies and working groups. These include cardiopulmonary exercise testing, which demonstrates abnormal responses in established pulmonary hypertension [14, 15]. Biomarkers, clearly, would be an appealing and less resource-intensive approach. To date, no biomarker has proven value as a reliable tool to detect early $\mathrm{PAH}$ [16], although in systemic sclerosis, a combination of $\mathrm{N}$-terminal pro-brain natriuretic peptide and pulmonary function tests may have utility [17, 18], as may the use of a predictive model based on a combination of oximetry and pulmonary function tests as part of a screening strategy in these patients [19]. The first, very preliminary report of a potential role for microRNA analysis has been recently published [20] and it is likely further reports will follow. Many of these approaches are discussed in a recent excellent review [21].

Supportive as we should be of "breathlessness is not OK" campaigns, they will lead to increasing referrals to our clinics and this will be a major challenge for which we must prepare by exploring these potential tools. We urgently need new tools to help us detect early pulmonary vascular disease in symptomatic patients where there is clinical suspicion of $\mathrm{PAH}$ but initial tests fail to confirm the diagnosis of PAH.

The study by PAVELESCU et al. [7] exploring the use of $\alpha$ suggests another potential tool to explore and highlights the need for novel approaches to ensure effective application of investigative tools across multiple centres. The study designers are to be congratulated for their innovative and thought provoking reports $[7,8]$.

The glory of overcoming these obstacles is that developing investigative strategies allowing earlier diagnosis and treatment protects the pulmonary vascular bed from progressive and crippling obliterative vasculopathy.

\section{References}

1 Humbert M, Coghlan GJ, Khanna D. Early detection and management of pulmonary arterial hypertension. Eur Respir Rev 2012; 21: 306-312.

2 Benza RL, Miller DP, Barst RJ, et al. An evaluation of long-term survival from time of diagnosis in pulmonary arterial hypertension from the REVEAL registry. Chest 2012; 142: 448-456.

3 Humbert M, Sitbon O, Chaouat A, et al. Pulmonary arterial hypertension in France: results from a national registry. Am J Respir Crit Care Med 2006; 173: 1023-1030.

4 Badesch DB, Raskob GE, Elliott CG, et al. Pulmonary arterial hypertension: baseline characteristics from the REVEAL registry. Chest 2010; 137: 376-387.

5 Kovacs G, Berghold A, Scheidl S, et al. Pulmonary arterial pressure during rest and exercise in healthy subjects: a systematic review. Eur Respir J 2009; 34: 888-894.

6 Naeije R, Vanderpool R, Dhakal BP, et al. Exericise-induced pulmonary hypertension. Am J Respir Crit Care Med 2013; 187: 576-583.

7 Pavelescu A, Vanderpool R, Vachiery JL, et al. Echocardiography of pulmonary vascular function in asymptomatic carriers of BMPR2 mutations. Eur Respir J 2012; 40: 1287-1289.

8 Grünig E, Weissman S, Ehlken N, et al. Stress Doppler echocardiography in relatives of patiens with idiopathic and familial pulmonary arterial hypertension. Results of a multi-center European analysis of pulmonary artery response to exercise and hypoxia. Circulation 2009; 119: 1747-1757.

9 Reeves JT, Linehan JH, Stenmark KR. Distensibility of the normal human lung circulation during exercise. Am J Physiol Lung Cell Mol Physiol 2005; 288: L419-L425.

10 Argiento $\mathrm{P}$, Chesler N, Mulè M, et al. Exercise stress echocardiography for the study of the pulmonary circulation. Eur Respir J 2010; 35: 1273-1278.

11 Sanz J, Kariisa M, Dellegrottaglie S, et al. Evaulation of pulmonary artery stiffness in pulmonary hypertension with cardiac magnetic resonance. JACC Cardiovasc Imaging 2009; 2: 286-295.

12 Luke HS, Grapsa J, Dawson D, et al. Echocardiographic assessment of pulmonary hypertension: standard operating procedure. Eur Respir Rev 2012; 21, 125: 239-248. 
13 Saggar R, Sitbon O. Hemodynamics in pulmonary arterial hypertension: Current and future perspectives. Am J Cardiology 2012; 110: 9S-15S.

14 Sun XG, Hansen JE, Oudiz RJ, et al. Exercise pathophysiology in patients with primary pulmonary hypertension. Circulation 2001; 104: 429-435.

15 Tolle JJ, Waxman AB, Van Horn TL, et al. Exercise induced pulmonary arterial hypertension. Circulation 2008; 118: 2183-2189.

16 Cracowski JL, Leuchte HH. The potential of biomarkers in pulmonary arterial hypertension. Am J Cardiol 2012; 110: 32S-38S.

17 Allanore Y, Borderie D, Avouac J, et al. High N-terminal pro-brain naturetic peptide levels and low diffusing capacity for carbon monoxide as independent predictors of the occurrence of pre-capillary pulmonary arterial hypertension in patients with systemic sclerosis. Arth Rheum 2008; 58: 284-291.

18 Thakkar V, Stevens WM, Prior D, et al. N-terminal pro-brain natriuretic peptide in a novel screening algorithm for pulmonary arterial hypertension in systemic sclerosis: a case-control study. Arthritis Res Ther 2012; 14: R143.

19 Schreiber BE, Valerio CJ, Keir GJ, et al. Improving the detection of pulmonary hypertension in systemic sclerosis using pulmonary function tests. Arth Rheum 2011; 63: 3531-3539.

20 Wei C, Henderson H, Spradley C, et al. Circulating miRNAs as potential marker for pulmonary hypertension. PLoS One 2013; 8: e64396.

21 Lau EMT, Manes A, Celermajer DS, et al. Early detection of pulmonary vascular disease in pulmonary arterial hypertension: time to move forward. Eur Heart J 2011; 32: 2489-2498. 